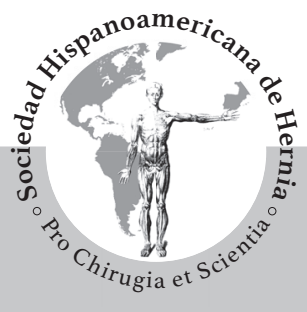

\title{
Original
}

\section{Controversias actuales en el tratamiento de la pseudohernia}

\section{Current management in the treatment of pseudohernia}

\section{Alfredo Moreno-Egea ${ }^{1,2}$}

1 Jefe de la Clínica Hernia. Hospital La Vega, Murcia (España)

${ }^{2}$ Profesor de Anatomía Quirúrgica. Departamento de Anatomía, Facultad de Medicina. Universidad Católica de San Antonio, Murcia (España)

Recibido: 23-06-2017

Aceptado: 23-06-2017

\section{Palabras clave:}

Pseudohernia, hernia lumbar, atrofia muscular, tomografía, cirugía abierta, doble malla.

\section{Resumen}

Introducción. Todavía no disponemos de ninguna evidencia para recomendar el tratamiento quirúrgico de la pseudohernia, debilidad fascial por atrofia muscular tras denervación. El objetivo del presente estudio es analizar los resultados de la cirugía en la pseudohernia mediante una técnica de doble reparación protésica, evaluando los posibles beneficios locales y generales para el paciente.

Métodos. Estudio clínico, prospectivo y descriptivo, en 12 pacientes operados por pseudohernia. La presencia de hernia es descartada mediante tomografía previa. El criterio principal de valoración fue la evaluación de la pared abdominal mediante medición del perímetro abdominal. Los criterios de valoración secundarios fueron los siguientes: morbilidad, satisfacción y datos del seguimiento.

Resultados. La cirugía mejoró significativamente la debilidad abdominal $(\mathrm{p}<0.05)$, sin morbilidad significativa, con una satisfacción superior al $80 \%(\mathrm{p}<0.05)$ y una significativa mayor calidad de vida $(\mathrm{p}<0.001)$.

Conclusiones. La pseudohernia puede repararse mediante la técnica de doble malla, con una morbilidad mínima. Esta técnica ofrece buenos resultados locales y una mejor calidad de vida en pacientes bien seleccionados y motivados.

\section{Abstract}

Background. There is still no evidence to recommend surgery in pseudohernia, fascial weakness by muscle atrophy after denervation. The aim of this study is to analyze the results of surgery in pseudohernia by double prosthetic repair technique, evaluating potential local and general benefits to the patient.

Methods. Prospective and descriptive clinical study in 12 patients operated by pseudohernia. A CT-scan was required to rule hernia. The primary endpoint was the assessment of the abdominal wall by measuring waist circumference. The secondary endpoints were morbidity, satisfaction and follow-up data.

Results. Surgery significantly improve abdominal weakness $(\mathrm{p}<0.05)$, with no significant morbidity, with satisfaction over $80 \%(\mathrm{p}<0.05)$ and increased quality of life $(\mathrm{p}<0.001)$.

Conclusions. The pseudohernia can be repaired by a double mesh technique with minimal morbidity. This technique obtains good local results and a better quality of life, in patients well selected and motivated.

* Autor para correspondencia. Dr. A. Moreno Egea. Avda. Primo de Rivera 7, 5. D. D. 3008. Murcia (España). Teléfono: $968-905061$. Fax: 968 232484. Correo electrónico: morenoegeaalfredo@gmail.com 


\section{Introduccion}

Las incisiones quirúrgicas pueden causar lesión nerviosa, lo que conduce a una progresiva atrofia muscular y debilidad de la pared abdominal ${ }^{1,2}$. En este caso, los pacientes pueden desarrollar un tumor en el área de la incisión, que clínicamente se comporta como una ruptura fascial. Sin embargo, en esos pacientes no hay hernia, no existe un anillo palpable causado por la rotura de los planos de la pared abdominal y demostrable mediante tomografía computarizada. Esta situación clínica particular se denomina $p s e u d o h e r n i a^{3-6}$. La incidencia de esta complicación quirúrgica puede oscilar entre el 10-30 \%7.

Hoy en día no disponemos de documentación suficiente para poder justificar una operación en pacientes con una pseudohernia confirmada mediante prueba de imagen. Las controversias que plantea este tema son la necesidad de cirugía o no, el tipo de abordaje posible, la técnica que se debe realizar, el uso (o no) de malla, el beneficio real para la función y fisiología de la pared abdominal y la posible mejora de los síntomas. Todas estas cuestiones son todavía interrogantes sin respuesta. Además, recientemente, la pared abdominal está adquiriendo una mayor importancia clínica como elemento indispensable para la contención uniforme del contenido abdominal y soporte muscular. Traumatólogos y fisioterapeutas han enfatizado su interés centrado en la fascia toracolumbar y la teoría del core, de forma que la lesión de la pared abdominal, sea por rotura o debilidad, podría generar dolor de espalda, agravar el estreñimiento, la incontinencia, ocasionar problemas para caminar, etc..$^{8-11}$.

Este estudio realiza una extensa revisión bibliográfica sobre el tema y analiza si la reparación de doble malla en la pseudohernia proporciona una nueva tensión muscular a la pared abdominal, permitiendo restaurar una adecuada contención abdominal y, en consecuencia, mejorar la calidad de vida de estos pacientes.

\section{Pacientes y métodos}

Se presenta un estudio clínico prospectivo, descriptivo y unicéntrico, realizado entre enero de 2000 y mayo de 2015 en 14 pacientes diagnosticados de pseudohernias. Todos los pacientes fueron evaluados en una unidad multidisciplinaria especializada en la pared abdominal, donde se realizaba una historia clínica detallada, un examen físico completo y una tomografía computarizada, con reconstrucción de la pared abdominal posterolateral.

Estos pacientes fueron seguidos de forma personalizada entre $1 \mathrm{y}$ 3 años de forma ambulatoria para optimizar su salud, utilizando las siguientes pautas: no fumar durante al menos un año, control de peso, rehabilitación y fisioterapia y establecimiento de estilos de vida saludables. Después de una información detallada sobre el procedimiento, las posibilidades y los riesgos de la cirugía, los pacientes que aceptaron la operación y firmaron el formulario de consentimiento adecuado fueron incluidos en el presente estudio. El plan de investigación fue presentado y aprobado por el Comité de Ética. Se utilizó una base de datos (Microsoft Excel, Microsoft Corporation, Seattle, EE. UU.) para el seguimiento de todos los pacientes inscritos.

\section{Técnica quirúrgica}

La operación era siempre realizada por el mismo cirujano, de forma estandarizada (AME) y bajo anestesia general endotra- queal. El paciente era colocado en decúbito lateral con el elevador tipo renal para quebrar la mesa a nivel lumbar. La reparación era una doble reconstrucción protésica y aponeurótica, publicada previamente. Se resecaba la cicatriz y se disecaban dos colgajos amplios de piel y tejido subcutáneo mediante electrocauterización. Se colocaba una malla (TiMesh $\AA$, pfm Medical Ag., Colonia, Alemania) en el espacio preperitoneal para solapar ampliamente todo el espacio diseccionado y se fijaba con

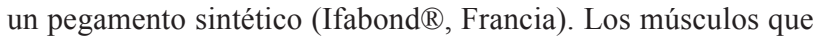
formaban los límites del defecto, oblicuo interno y transverso, se aproximaban y suturaban sobre la primera malla. Después se colocaba una segunda malla de igual material a nivel intermuscular (oblicuo interno-oblicuo externo) y se fijaba igualmente con adhesivo. Sobre esta segunda reparación fascial se aproximaba el plano muscular correspondiente al oblicuo externo. Se dejaba un drenaje en el tejido celular subcutáneo (dependiendo de la cantidad de tejido disecado y del espacio muerto resultante) y se retiraba cuando el débito era menor de $50 \mathrm{ml} /$ día. Los colgajos laterales de piel y tejido subcutáneo se aproximaban cuidadosamente para evitar espacios muertos (subcutáneo con sutura continua absorbible $2 / 0$ y piel con grapas).

\section{Seguimiento}

Los pacientes fueron dados de alta cuando presentaban el dolor controlado y el equipo quirúrgico estaba satisfecho con la ausencia de complicaciones inmediatas. Todos los pacientes fueron incluidos en un protocolo de seguimiento y revisados en consulta externa del centro a los 1, 3, 6 y 12 meses.

\section{Objetivo principal y secundario}

El objetivo principal del estudio era evaluar la calidad de la pared abdominal, definida y cuantificada por dos parámetros:

1) Evaluación clínica. Cuantificación del perímetro abdominal, medido antes y después de la cirugía, con una cinta métrica milimétrica.

2) Evaluación del bienestar, cuantificado con una encuesta de satisfacción del paciente tipo Liker, que calificaba entre $0 \mathrm{y}$ 1 (0: no satisfecho, 1: satisfecho).

Como objetivo secundario se evaluaron los datos clínicos de los pacientes (edad, comorbilidad, cirugía previa, etc.) y su morbilidad (seroma, hematoma, infección y dolor).

\section{Definiciones de variables}

El seroma era definido como aquella colección de fluidos detectados por palpación al examen clínico. La infección de la herida era definida como el enrojecimiento, descarga de pus de la herida o mediante un cultivo positivo de bacterias. La recurrencia del tumor o bulto parietal se valoraba a los 12 meses mediante examen clínico y tomografía computarizada. La calidad de vida de los pacientes se cuantificaba utilizando una escala estándar modificada, el EuroQol$5 \mathrm{D}$, considerando los valores 0 (no satisfecho) y 1 (satisfecho). El 
Tabla 1. Datos clínicos y demográficos de los

pacientes con atrofia muscular por denervación tras cirugía

\begin{tabular}{lc}
\multicolumn{1}{c}{ Datos demográficos } & $(\mathrm{n}=14)$ \\
\hline Edad (años) & $63.4 \pm 9.6$ \\
Sexo & \\
$\quad$ Hombre & $6(42.9)$ \\
Mujer & $8(57.1)$ \\
IMC (kg/m2) & $31.4 \pm 7.8$ \\
Comorbilidad & \\
Fumador & $8(66.6)$ \\
Diabetes melitus & $5(41.7)$ \\
Hipertension arterial & $7(58.3)$ \\
Cirugía previa & \\
$\quad$ Nefrectomía & \\
Vascular & $9(64.2)$ \\
Columna & $3(21.5)$ \\
\hline
\end{tabular}

Características clínicas de los pacientes con atrofia muscular. Los datos son expresados en valor absoluto (porcentaje) y mediana \pm DS

(IMC: índice de masa corporal)

rango de seguimiento fue de 14-45 meses (media: 24 meses) y se completó a un año en el $100 \%$ de los pacientes.

\section{Análisis estadístico}

Los resultados se expresan como mediana \pm DS y en valor absoluto (porcentaje). El análisis se realizó utilizando el paquete estadístico para ciencias sociales (SPSS) para Windows (SPSS Inc., v15.0, Chicago, EE. UU.). Todos los datos fueron procesados por un observador independiente.

\section{Resultados}

Las características clínicas de los pacientes se presentan en la tabla 1 (fig. 1). El índice de masa corporal promedio fue de 31.8 $\pm 7.5 \mathrm{~kg} / \mathrm{m}^{2}$. Hubo un predominio de mujeres (57.1\%). Los 14 pacientes habían sido sometidos a cirugía abdominal ipsilateral entre 2 y 20 años antes de la tomografía computarizada inicial. En la tomografía, todos los pacientes presentaban lesión por denervación de la pared abdominal posterolateral, pero con todas las capas musculares intactas (sin hernia). La atrofia se limitó al grupo muscular lateral (oblicuo externo e interno, y transverso abdominal) en diez pacientes y en grupos anterolaterales de músculos abdominales (más recto abdominal) en cuatro pacientes (fig. 2). La cirugía inicial que generó el tumor parietal fue de origen urológico en nueve pacientes, vascular en tres y en el acceso de la columna vertebral en otros dos.

No se registraron complicaciones intraoperatorias derivadas del procedimiento. Se colocaron dos mallas, preperitoneal y intermuscular, en todos los pacientes. El tiempo quirúrgico medio fue
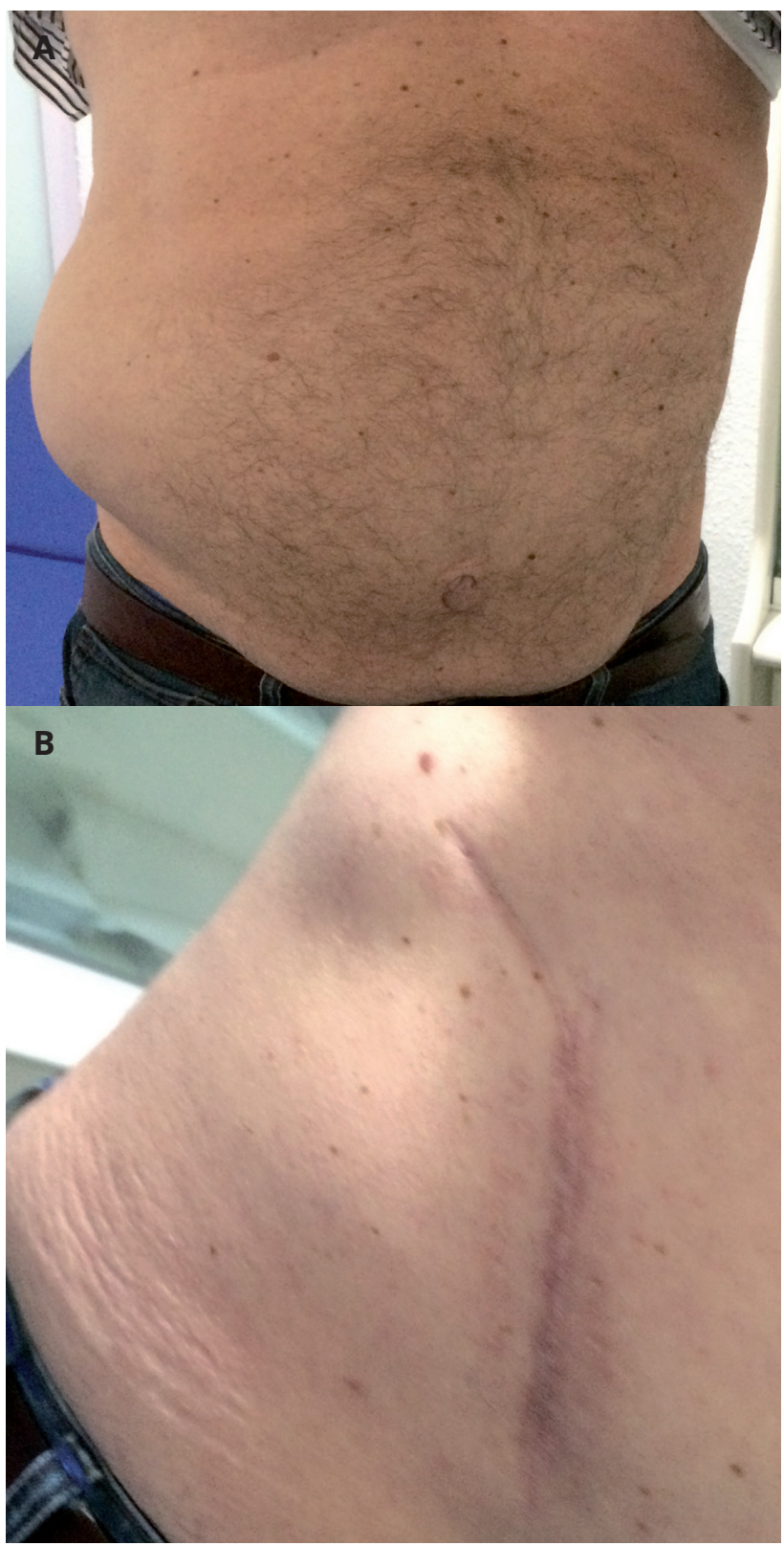

Figura 1. A. Pseudohernia: defecto estético de un paciente tras cirugía renal (nefrectomía). A: Se muestra una clara asimetría abdominal en el flanco derecho. B: Se aprecia el detalle de la incisión previa.

de $84 \pm 16$ minutos, y la estancia media hospitalaria, de $4 \pm 2$ días. No hubo infecciones del sitio quirúrgico, ni superficiales ni profundas. Las únicas complicaciones posoperatorias registradas fueron la presencia de hematomas y de seromas, que se resolvieron espontáneamente antes de 3 meses (tabla 2).

No hubo reoperaciones durante el seguimiento. No hubo recurrencias al año de la cirugía. Dos pacientes desarrollaron dolor parietal local, que se resolvió dentro de los 8 meses siguientes a la operación. A pesar de ello, ningún paciente precisó tratamiento analgésico tras los primeros 3 meses. Todos los pacientes recuperaron su actividad normal. El tiempo medio de seguimiento fue de $24 \pm 12$ meses (rango: 14-45 meses). 


\begin{tabular}{lc}
\multicolumn{2}{c}{ Tabla 2. Datos operatorios y de control } \\
posoperatorio
\end{tabular}

Los datos son presentados como valor absoluto (porcentaje) y mediana \pm DS (IO: intraoperatorio; PO: posoperatorio) $\star p<0.05(p=0.002)$

Todos los parámetros evaluados en la encuesta de calidad de vida fueron estadísticamente significativos a favor de la cirugía (figs. 3 y 4). La reparación de pseudohernia condujo a una mejora en calidad de vida, satisfacción corporal, incontinencia urinaria y estreñimiento $(\mathrm{p}<0.001)$ (tabla 3$)$.

\section{Discusión}

En la bibliografía publicada solo hay dos trabajos específicos sobre el tratamiento de la pseudohernia (tabla 4). Ambos analizan el problema desde un punto de vista exclusivamente estético ${ }^{12,13}$. Nuestro estudio es el primero que valora si la reparación de la pseudohernia ofrece un beneficio en la calidad de vida a los pacientes que la padecen. La hipótesis del estudio se hace eco de las nuevas teorías que consideran a la fascia toracolumbar y a los músculos abdominales posterolaterales como parte de una estructura general del organismo que integra el suelo pélvico y la región toracolumbar. Por lo tanto, podríamos sugerir que la reparación de la pared abdominal posterolateral, al restaurar la tensión muscular abdominal y aumentar la contención, podría mejorar algunos síntomas como el dolor de espalda, el estreñimiento o la incontinencia de los pacientes que presentan dicha debilidad muscular ${ }^{8-10}$.

Aunque la cirugía ha avanzado mucho en los últimos años, la posibilidad de lesión nerviosa y de pseudohernia sigue siendo una complicación frecuente y tiene un gran interés clínico para las personas que lo padecen ${ }^{1-7}$. También se han publicado como causa de pseudohernia el abordaje laparoscópico y las incisiones para eliminar piezas resecadas de tumores ${ }^{14}$. De esta manera, sabemos que la laparoscopia no evita esta complicación, por

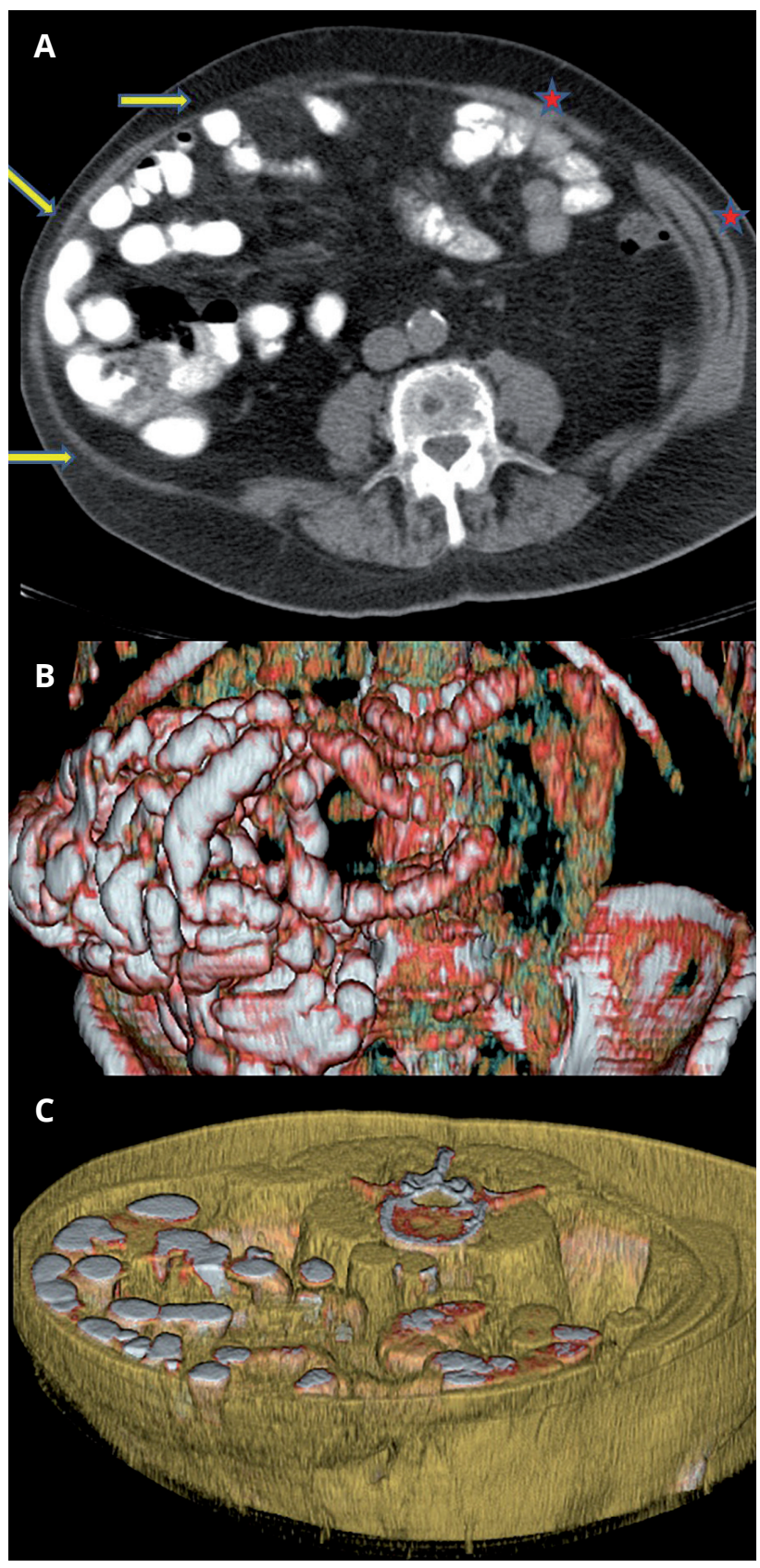

Figura 2. Estudio radiológico de un paciente con pseudohernia. A: Debilidad de la pared abdominal anterolateral izquierda con protrusión de asas intestinales (Estrella: musculatura normal; Flecha: atrofia muscular). B-C: Reconstrucción de la pared abdominal, demostrando el defecto lateral.

lo que vuelve a generar el debate de su abordaje mínimamente invasivo.

\section{¿Cuándo se debe operar?}

La necesidad de operar si no existe defecto fascial es uno de los puntos más controvertidos de este padecimiento. Autores 


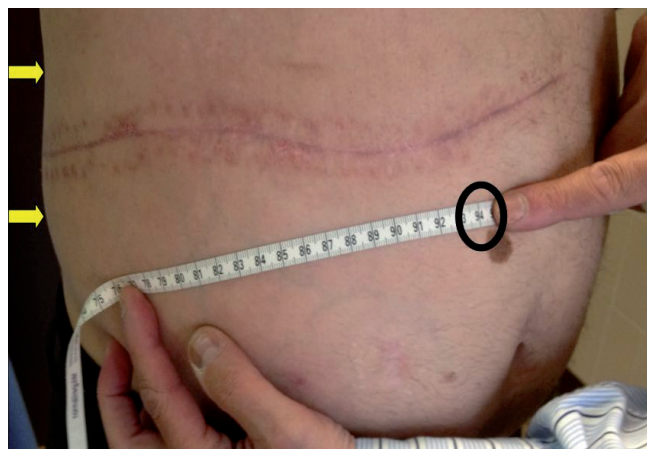

Figura 3. Control posoperatorio. El paciente muestra mayor contención abdominal y menos dolor local. como Petersen aconsejan que solo los pacientes con una verdadera hernia del flanco, con bordes palpables, sean candidatos para la reparación con malla ${ }^{5}$. En contraste, Staerman o Pineda creen que las principales razones para indicar la cirugía son la presencia de molestias locales y la apariencia ${ }^{3,13}$. La indicación de la cirugía por razones estrictamente estéticas debe considerarse con mucha cautela. No es fácil atribuir el dolor de espalda, o cualquier otro síntoma inespecífico, solo a la debilidad de la pared abdominal posterolateral. Por tanto, el estudio preoperatorio debe ser meticuloso, con un buen estudio preoperatorio para planificar adecuadamente las ventajas potenciales de la operación.

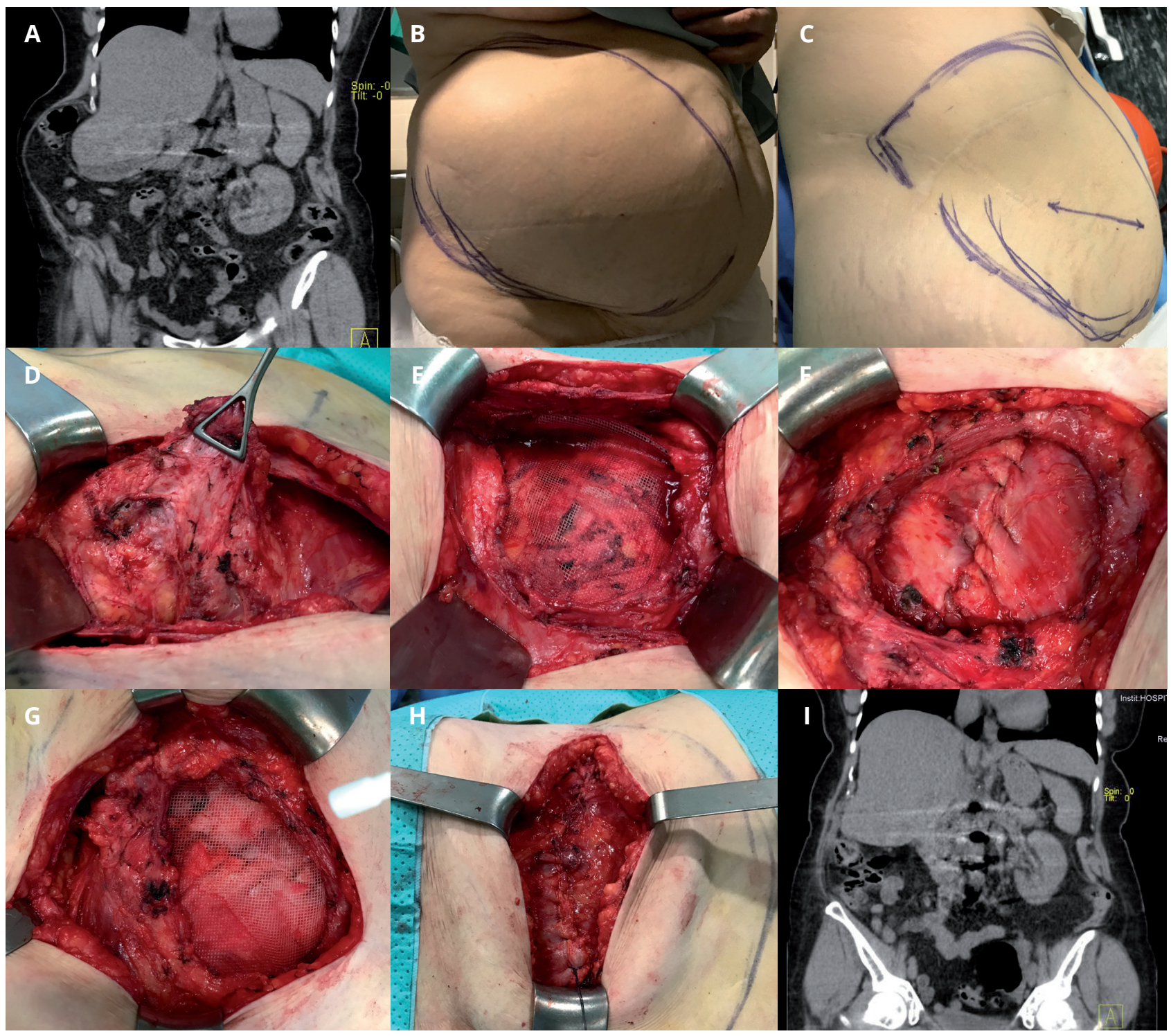

Figura 4. Mujer de 73 años con pseudohernia tras cirugía renal. A: Tac preoperatorio que confirma la atrofia muscular sin hernia. B-C: Exploración clínica (bulto posterolateral). D: Debilidad muscular resultado de la lesión nerviosa. E: Primera malla, situada en el plano preperitoneal, profunda al músculo transverso abdominal. F: Plicatura del músculo transverso abdominal y del oblicuo interno en el sentido de sus fibras. G: Segunda malla, situada en el plano intermuscular, profunda al músculo oblicuo externo. H: Plicatura del músculo oblicuo externo en el sentido de sus fibras (cuarto plano de reconstrucción). I: Control de tac al año, que muestra una recuperación del grosor y de la tensión parietal. 


\begin{tabular}{|c|c|c|c|}
\hline & $\begin{array}{c}\text { Preop. } \\
(n=12) \\
0-1\end{array}$ & $\begin{array}{c}\text { Posop. } \\
(n=12) \\
0-1\end{array}$ & $\begin{array}{c}\mathrm{p} \\
\text { valor }\end{array}$ \\
\hline Mobilidad & $12-0$ & $0-12$ & 0.001 \\
\hline Autocuidado & $12-0$ & $0-12$ & 0.001 \\
\hline Actividades usuales & $12-0$ & $0-12$ & 0.001 \\
\hline Dolor/incomodidad & $12-0$ & $2-10$ & 0.001 \\
\hline Ansiedad/depresion & $10-2$ & $4-8$ & 0.001 \\
\hline Satisfaction corporal & $12-0$ & $1-11$ & 0.001 \\
\hline $\begin{array}{l}\text { Incontinencia } \\
\text { urinaria }\end{array}$ & $7-5$ & $3-9$ & 0.001 \\
\hline Estreñimiento & $8-4$ & $2-10$ & 0.001 \\
\hline
\end{tabular}

Valores expresados como frecuencias (EuroQol-5D:

0 : No satisfecho o cambios mínimos; 1: Satisfecho)

En la bibliografía se han publicado casos de pacientes operados sin un conocimiento previo de la existencia de una hernia. Salameh y Yavuz recomiendan realizar una tomografía para evitar la operación en aquellos casos en que no exista una hernia (rotura fascial de algún plano parietal) ${ }^{15-17}$. Nosotros creemos que la indicación quirúrgica debe basarse en un diagnóstico preoperatorio muy preciso. La existencia de un defecto fascial debe conocerse antes de proponer una reparación quirúrgica. Además, antes de planificar la operación debe conocerse la localización exacta del defecto o debilidad en relación con el espacio lumbar y el estado de los músculos laterales. Por tanto, consideramos que la TC debe realizarse siempre, en pacientes con cirugía previa y protuberancia en el flanco ${ }^{3,18}$.

\section{¿Qué abordaje?}

El enfoque de una pseudohernia genera mucha confusión en la bibliografía publicada. Algunos autores prefieren un abordaje directo sobre el bulto ${ }^{5,13,14}$. Esta actitud permite eliminar la cicatriz y evaluar la pared abdominal directamente, pero también significa la disección en un campo traumatizado. Otros autores, como Zieren, prefieren realizar un abordaje a través de una laparotomía media, alcanzando la debilidad desde un plano normal no dañado y evitando las cicatrices ${ }^{20}$.

Para complicar aún más la decisión, otros autores, como Hoffman y cols., aconsejan realizar la operación a través de una incisión de abdominoplastia ${ }^{12}$. Esta opción parece inicialmente más agresiva, pero permite un mejor acceso a la pared abdominal total, para realizar posteriormente una adecuada plicatura. Lo que es innegable es que la combinación de una abdominoplastia (realizada por cirujanos bien entrenados y experimentados) proporciona unos beneficios psicológicos que no deberían subestimarse en pacientes bien seleccionados ${ }^{21}$. Además, aumenta la motivación y promueve la cooperación de los pacientes durante el período de seguimiento. Por otra parte, la mayor agresión podría causar un aumento de la morbilidad y de la tasa de recurrencia, ya que obliga a una reparación superficial (no preperitoneal o intermuscular). Creemos que un abordaje directo tiene más ventajas que desventajas, ya que permite evaluar cada plano fascial y muscular, extender la disección medial o dorsal y realizar una plicatura parietal, creando una tensión totalmente controlada ${ }^{22}$.

\section{¿Qué técnica?}

¿Puede el abordaje laparoscópico reducir el tumor abdominal? ¿La fibrosis y la retracción producidas por la malla intraperitoneal mejoran la calidad de la pared abdominal? No hay evidencia para responder a estas preguntas. Todavía no está claro si un abordaje laparoscópico puede lograr una remodelación de la pared abdo-

\begin{tabular}{|c|c|c|c|c|c|c|c|}
\hline & Indicación & $\mathrm{TC}$ & $\mathrm{Ab}$. & Técnica & Malla & $\mathrm{R}$ & $\mathrm{S}$ \\
\hline Bolkier (1991) & Hernia & No & Local & Plicatura fascial & No & - & - \\
\hline Staerman (1997) & $\begin{array}{l}\text { Hernia } \\
\text { Ps (2) }\end{array}$ & No & Local & Sutura retención & No & No & 14 \\
\hline Petersen (2002) & Hernia & No & Local & - & Preperitoneal & No* & 33 \\
\hline Hoffman (2004) & Ps (3) & Sí & AP & Plicatura & Onlay & $1(33)$ & 5 \\
\hline Zieren (2007) & Hernia & No & LM & - & Preperitoneal & $\mathrm{No} * *$ & 60 \\
\hline Pineda (2013) & Ps (8) & No & Local & Flap IO & Intermuscular & 2 & 12 \\
\hline Dumanian (2016) & $\begin{array}{l}\text { Hernia } \\
\text { Ps (4) }\end{array}$ & Sí & Local & Plicatura muscular & Intermuscular & - & 27 \\
\hline Moreno-Egea (2016) & Ps (14) & Sí & Local & Doble plicatura & $\begin{array}{l}\text { Preperitoneal } \\
\text { Intermuscular }\end{array}$ & No & 64 \\
\hline
\end{tabular}

Ps: Pseudohernia; TC: Tomografía; Ab.: Abordaje; FT: Fascia transversalis; AP: Abdominoplastia; LM: Laparotomia media; R: Recurrencia; S: Seguimiento (en meses); *: Dolor persistente (75 \%); **: $100 \%$ recurrencias en el grupo de incisión local (7/7). 
minal, como se puede conseguir mediante un abordaje abierto. La laparoscopia tiene ventajas como el abordaje mínimamente invasivo, pero plantea problemas al trabajar con un neumoperitoneo (estado no fisiológico de trabajo) y no poder controlar una posible plicatura de la pared abdominal. Por lo tanto, autores como Petersen dudan de la función de esta técnica, incluso en casos de hernias ${ }^{5}$. En la pseudohernia, Purnell y cols. proponen la resección de tejidos dañados y una plicatura, que no se puede realizar por vía laparoscópica. En la hernia lumbar, la laparoscopia sí podría ser una técnica eficaz, pero cuando el defecto o la laxitud implica completamente a toda la pared abdominal posterolateral, ¿puede tener indicación? En 2005, Tobias-Machado y cols. publicaron los resultados de siete pacientes con hernias lumbares tratados por vía laparoscópica, y admitieron que el paciente que presentaba atrofia muscular no consiguió un resultado estético satisfactorio ${ }^{25}$. La experiencia del autor en un estudio previo confirmaba que el abordaje laparoscópico no ofrecía ningún beneficio en el tratamiento de la pseudohernia lumbar. La baja morbilidad del abordaje laparoscópico no puede usarse como argumento para justificar el empleo de esta técnica en los pacientes con pseudohernias ${ }^{5}$.

\section{¿Qué malla, de qué tamaño y cuántas?}

Hoy en día no podemos entender la reparación de una hernia sin malla. Pero ¿qué sucede si no hay defecto aponeurótico? ¿Es necesaria una malla? ¿En qué plano de la pared abdominal debe ser colocada? Si ponemos dos mallas, ¿aumentarán las dos mallas la continencia abdominal? No tenemos suficiente documentación para responder a estas cuestiones. La controversia respecto a la necesidad del uso de una malla o no permanece en suspense. Algunos autores usan PP, otros PTFE, y también hay quien defiende el uso de una malla biológica, a pesar de su elevado coste ${ }^{28}$.

Aún mayor es la controversia respecto al tamaño de la malla, la fijación, el plano donde debe situarse y la necesidad de una segunda malla $^{29}$. Purnell y cols. aconsejan utilizar una malla pequeña que no sea fijada a estructuras óseas para evitar una rigidez excesiva $(\text { tabla } 5)^{23}$. Las desventajas de la operación de Dumanian son el uso de múltiples suturas transmurales, lo que incrementa el riesgo de dolor, y el limitado tamaño de la malla que aconseja (no mayor de $7 \mathrm{~cm}$ ), lo que incrementa el riesgo de recurrencia, al no proteger ampliamente toda la pared denervada y atrófica. Dicho autor repara el defecto como si fuera una hernia, a diferencia de nuestra propuesta que considera a la pseudohernia como un problema muscular global, sin claros límites ${ }^{23}$. En nuestra experiencia, el concepto de nuestra operación basado en una reconstrucción parietal (más que el de una reparación focal) evita estas desventajas y mejora los resultados a largo plazo, como se demuestran en el caso de la paciente de la fig. 4: el estudio radiológico de control informa que la paciente, tras la operación, consigue un mayor grosor de la pared muscular lateral derecha, siendo antes una lámina delgada donde en algunas zonas no se identificaban sus distintas capas y ahora alcanza un grosor superior a $11 \mathrm{~mm}$, objetivándose claramente su estratificación. Esta recuperación de la tensión muscular puede justificar el cambio de la morfología abdominal, más aplanada antes de la cirugía, con un eje transversal previo de $35.6 \mathrm{~cm}$ (y tras la operación, de $33.6 \mathrm{~cm}$ ) y con un mayor diámetro anteroposterior que ha pasado de $24 \mathrm{~cm}$ a $27 \mathrm{~cm}$. En conclusión, se consigue la recuperación del tono y grosor de pared muscular abdominal derecha.

\begin{tabular}{|c|c|c|}
\hline & Dumanian & Moreno-Egea \\
\hline Tac & No & Sí \\
\hline Reconstrucción & 3 planos & 4 planos \\
\hline Abordaje & $\begin{array}{l}\text { Intraabdominal o } \\
\text { intermuscular }\end{array}$ & $\begin{array}{l}\text { Preperitoneal } \\
\text { más } \\
\text { intermuscular }\end{array}$ \\
\hline $\begin{array}{l}\text { 1) Plano muscular } \\
\text { TA-OI }\end{array}$ & $\begin{array}{l}\text { Plicatura o } \\
\text { resección }\end{array}$ & $\begin{array}{l}\text { Plicatura del } \\
\text { transverso }\end{array}$ \\
\hline Malla & 1 pequeña $(7 \mathrm{~cm})$ & $\begin{array}{l}2 \text { grandes } \\
(20-15 \mathrm{~cm})\end{array}$ \\
\hline Fijación & Sutura transmural & $\begin{array}{l}\text { 4-6 suturas más } \\
\text { pegamento }\end{array}$ \\
\hline $\begin{array}{l}\text { 2) Plano muscular } \\
\text { OE }\end{array}$ & Suturas en 8 & $\begin{array}{l}\text { Plicatura del } \\
\text { oblicuo }\end{array}$ \\
\hline Diseño & Local & Integral \\
\hline
\end{tabular}

TA: Músculo transverso abdominal; OE: Músculo oblicuo externo; Ol: Músculo oblicuo interno.

Por el contrario, Petersen y cols. defienden el uso de una malla grande y con una fijación intensa mediante suturas transfasciales ${ }^{5}$. La mayoría de autores recomiendan colocar la malla en un plano profundo de la pared abdominal, pero Hoffman y cols. la sitúan en el plano superficial (supraaponeurotico) ${ }^{12}$. Zieren y cols. aconsejan que la malla sea de gran tamaño y alcance el músculo contralateral para asegurar mayor tensión y disminuir la posibilidad de recurrencia $^{20}$. Después de la revisión bibliográfica, no podemos concluir nada con evidencia. El autor ha estado utilizando una reparación con doble malla durante más de 10 años, en hernias complejas del flanco y con buenos resultados, y la experiencia que se presenta en este trabajo es la mayor que se ha documentado en la pseudohernia hasta la fecha. El uso de dos mallas nos ofrece la posibilidad de generar una mayor fibrosis a dos niveles distintos de la pared abdominal adelgazada y disminuir la necesidad de fijación mecánica (suturas o grapas), y esto sin añadir morbilidad a la operación ${ }^{30}$.

\section{Conclusión}

Para el tratamiento de la pseudohernia, nosotros recomendamos la cirugía abierta como abordaje y la reconstrucción musculoaponeurótica bajo tensión controlada con doble malla como técnica. Este manejo quirúrgico ofrece, en pacientes bien seleccionados y motivados, buenos resultados locales y una mejor calidad de vida.

\section{Bibliografía}

1. Gardner GP, Josephs LG, Rosca M, Rich J, Woodson J, Menzoian JO. The retroperitoneal incision: an evaluation of postoperative flank "bulge". Arch Surg. 1994;129:753-756. 
2. Fahim DK, Kim SD, Cho D, Lee S, Kim DH. Avoiding abdominal flank bulge after anterolateral approaches to the thoracolumbar spine: Cadaveric study and electrophysiological investigation. J Neurosurg Spine 2011;15:532-540.

3. Staerman F, Staerman H, Guiraud P, Mhidia A, Guille F, Lobel B. Autogenous skin graft in the treatment of large incisional lumbar hernias and bulges. Eur Urol. 1997;32:209-212.

4. Chiu H-Y, Lin S-J. A painful bulge in the left flank. JAMA 2013;310(6):639-640.

5. Moreno-Egea A, Sánchez-Elduayen M, De Andrés EP, Carrillo-Alcaraz A. Is muscular atrophy a contraindication in laparoscopic abdominal wall defect repair? A prospective study. Am Surg. 2012;78(2):178184.

6. Petersen S, Schuster F, Steinbach F, Henke G, Hellmich G, Ludwig $\mathrm{K}$. Sublay prosthetic repair for incisional hernia of the flank. J Urol. 2002;168:2461-2463.

7. Plata-Bello J, Roldan H, Brage L, Rahy A, García-Marín V. Delayed abdominal pseudohernia in young patient alter lateral lumbar interbody fusion procedure: case report. World Neurosurg. $2016 \mathrm{Apr}$ 11. pii: S1878-8750(16)30083-3. DOI: 10.1016/j.wneu.2016.04.010. [Epub ahead of print].

8. Gatton ML, Pearcy MJ, Pettet GJ, Evans JH. A three-dimensional mathematical model of the thoracolumbar fascia and an estimate of its biomechanical effect. J. Biomech. 2010;43(14):2792-2797.

9. Vleeming A, Pool-Goudzwaard AL, Stoeckart R, van Wingerden JP, Snijders CJ. The posterior layer of the thoracolumbar fascia. Its function in load transfer from spine to legs. Spine. 1995;20(7):753-758.

10. Willard FH, Vleeming A, Shuenke MD, Danneels L, Schleip R. The thoracolumbar fascia: anatomy, function and clinical considerations. J Anat. 2012;221(6):507-536.

11. Barker PJ, Briggs CA, Bogeski G. Tensile transmission across the lumbar fasciae in un embalmed cadavers: effects of tension to various muscular attachments. Spine. 2004;29:129-138.

12. Hoffman RS, Smink DS, Noone RB, Noone RB Jr, Smink RD Jr. Surgical repair of the abdominal bulge: correction of a complication of the flank incision for retroperitoneal surgery. J Am Coll Surg. 2004;199(5):830-835.

13. Pineda DM, Rosato EL, Moore JH. Flank bulge following retroperitoneal incisions: A myofascial flap repair that relieves pain and cosmetic sequelae. Plast Reconstr Surg. 2013;132(1):181e-183e.

14. Bolkier M, Moskovitz B, Ginesin Y, Levin DR. An operation for incisional lumbar hernia. Eur Urol. 1991;20(1):52-53.

15. Durham-Hall A, Wallis S, Butt I, Shrestha BM. Abdominal wall pseudohernia following video-assisted thoracoscopy and pleural biopsy. Hernia. 2009;13(1):93-95.
16. Salameh JR, Salloum EJ. Lumbar incisional hernias: diagnostic and management dilemma. JSLS 2004;8(4):391-394.

17. Yavuz N, Ersoy YE, Demirkesen O, Tortum OB, Erguney S. Laparoscopic incisional lumbar hernia repair. Hernia 2009;13:281-286.

18. Goodman P, Balachandran S. Postoperative atrophy of abdominal wall musculature: CT demonstration. J Comput Assist Tomogr. 1991;15(6):989-993.

19. Moreno-Egea A, Carrillo-Alcaraz A. Management of non-midline incisional hernia by the laparoscopic approach: results of a long-term follow-up prospective study. Surg Endosc. 2012;26(4):1069-1078.

20. Zieren J, Menenakos C, Taymoorian K, Müller JM. Flank hernia and bulging after open nephrectomy: mesh repair by flank or median approach? Report of a novel technique. Int Urol Nephrol. 2007;39:989-993.

21. Moreno-Egea A, Campillo-Soto Á, Morales-Cuenca G. Does Abdominoplasty Add Morbidity to Incisional Hernia Repair? A Randomized Controlled Trial. Surg Innov. 2016 Apr 28. pii: 1553350616646480. [Epub ahead of print]

22. Moreno-Egea A, Alcaraz AC, Cuervo MC. Surgical options in lumbar hernia: laparoscopic versus open repair. A long-term prospective study. Surg Innov. 2013;20(4):331-344.

23. Purnell CA, Park E, Turin SY, Dumanian GA. Postoperative flank defects, hernias, and bulges: A reliable method for repair. Plast Reconstr Surg. 2016;137(3):994-1001.

24. Shekarriz B, Graziottin TM, Gholami S, Lu HF, Yamada H, Duh QY, et al. Transperitoneal preperitoneal laparoscopic lumbar incisional herniorrhaphy. J Urol. 2001;166:1267-1269.

25. Tobias-Machado M, Rincon FJ, Lasmar M, Zambon JP, Juliano RV, Wroclawski ER. Laparoscopic surgery for treatment of incisional lumbar hernia. Int Braz J Urol. 2005;31:309-314.

26. Forbes SS, Eskicioglu C, McLeod RS, Okrainec A. Meta-analysis of randomized controlled trials comparing open and laparoscopic ventral and incisional hernia repair with mesh. Br J Surg. 2009;96(8):851-8.

27. Sajid MS, Bokhari SA, Mallick AS, Cheek E, Baig MK. Laparoscopic versus open repair of incisional/ventral hernia: a meta-analysis. Am J Surg. 2009;197(1):64-72.

28. Pezeshk RA, Pulikkottil BJ, Bailey SH, Schaffer NE, Reece EM, Thornton NJ, et al. An evidence-based model for the successful treatment of flank and lateral abdominal wall hernias. Plast Reconstr Surg. 2015;136(2):377-385.

29. Baumann DP, Butler CE. Lateral abdominal wall reconstruction. Semin Plast Surg. 2012;26:40-48.

30. Moreno-Egea A. Double Prosthetic Repair for Complex Incisional Hernia Repair: Long-term Results and Evolution of the Technique. Am Surg. 2015;81(11):1138-1143. 